\title{
Templated growth of textured and luminescent \\ CaS:Eu thin films by a low-temperature solvothermal process
}

\author{
P F Smet and D Poelman \\ LumiLab, Department of Solid State Sciences, Ghent University, Krijgslaan 281-S1, \\ 9000 Gent (Belgium) \\ E-mail: philippe.smet@ugent.be
}

\begin{abstract}
The direct growth of luminescent sulfide thin films onto templated substrates was realised with a solvothermal synthesis method. The as-grown layers of SrS:Eu and CaS:Eu emit a bright and homogeneous photoluminescence, peaking at $610 \mathrm{~nm}$ and $650 \mathrm{~nm}$ respectively. $\mathrm{x}$-ray diffraction measurements showed that the layers grown at a temperature of only $200^{\circ} \mathrm{C}$ are strongly crystalline, with (200) out-ofplane orientation. Using silicon substrates, almost epitaxial growth could be obtained. Electron microscopy was used to study the growth, morphology and texture of the thin films. The main advantages of this innovative approach for the production of sulfide layers are the low-temperature process and scalability.
\end{abstract}

PACS numbers: 68.55.J-, 78.55.Et, 81.15.-z

Paper published in J. Phys.D: Appl. Phys. 2009, 42, 095306 


\section{Introduction}

Rare earth doped sulfides are an important class of luminescent materials. They have extensively been investigated for AC thin film electroluminescence (ACTFEL) [1], with the aim of finding efficient emitters for the three primary colours for full colour displays. This has resulted in a number of thin film phosphors [2], notably ZnS:Mn (yelloworange), CaS:Eu (red) and $\mathrm{BaAl}_{2} \mathrm{~S}_{4}: \mathrm{Eu}$ (blue). The latter material was found to be sufficiently efficient to use it as the sole phosphor in a full colour display, the green and red components being generated by downconversion of the blue $\mathrm{BaAl}_{2} \mathrm{~S}_{4}$ : Eu emission $[3]$.

These results have triggered the interest in the use of sulfide phosphors for application in wavelength-converted white-light emitting diodes (LEDs) [4]. As the efficiency of LEDs has increased tremendously over the last years, and is reaching 150 $\mathrm{lm} / \mathrm{W}[5]$, LEDs are becoming a viable alternative for incandescent and fluorescent lamps for general lighting applications [6]. One of the main issues - besides cost and heat dissipation - that currently prevents the widespread use of LEDs is their limited colour quality. Indeed, the usual approach for making white LEDs is to combine a blueemitting LED with YAG:Ce as a broad band yellow emitter which converts part of the blue light. This yields a whitish spectrum with a high correlated colour temperature of about $5600 \mathrm{~K}$ (corresponding to 'cold' white light) and a rather mediocre colour rendering index (CRI) of 73 [4], the main reason being the lack of red light. Because it is very difficult to obtain broad band red light emission from oxide phosphors, there is currently a large interest in red-emitting nitride [7] and sulfide phosphors [4]. Both types of phosphors use $\mathrm{Eu}^{2+}$ as a dopant. The $5 \mathrm{~d}-4 \mathrm{f}$ transition in $\mathrm{Eu}^{2+}$ is strongly influenced by the crystal field of the host; therefore, its emission can quite easily be shifted over almost the entire visible range.

The present work focuses on CaS:Eu as a saturated red emitter. This material 
is well known as a phosphor for thin film electroluminescence [9] and for wavelength conversion in LEDs [10]. The deposition of high quality sulfide thin films is not straightforward. Electron beam evaporation often leads to sulfur deficient thin films [11] and RF sputtering sulfides in a reproducible way is far from evident. In both cases, high deposition and/or annealing temperatures are required to obtain well-crystallized luminescent thin films and $\mathrm{H}_{2} \mathrm{~S}$ is needed. Hence, research efforts towards low cost and easily scalable deposition techniques are required. Furthermore, sulfides have a rather high refractive index (e.g. 2.1 for SrS [8]), which leads to optical outcoupling problems in thin film applications, as part of the light remains trapped due to total internal reflection. Consequently, the possibility of obtaining a textured thin film surface could be advantageous as well. Recently, Van Haecke et al. optimized a low temperature solvothermal synthesis method for $(\mathrm{Ca}, \mathrm{Sr}) \mathrm{S}: \mathrm{Eu}$ particles $[12,13]$. Depending on the synthesis conditions, regular octahedron-shaped particles are obtained, with size ranging from a few hunderd nanometers to a few microns. The method uses a low temperature $\left(\mathrm{T}_{\max }=200^{\circ} \mathrm{C}\right)$, does not involve toxic $\mathrm{H}_{2} \mathrm{~S}$ and presumably is easily scalable. In this paper we describe a modification of this method for the deposition of crystalline, textured and luminescent thin films.

\section{Experimental setup}

Undoped $\mathrm{CaS}$ and $\mathrm{SrS}$ thin films with a thickness of 20 to $50 \mathrm{~nm}$ were deposited by electron beam evaporation on single crystal silicon (100) wafers or vycor (Corning 1737 glass) and used as template layers. Then, the substrates with a typical size of 1 by 4.5 $\mathrm{cm}^{2}$ were vertically inserted in an autoclave together with a mixture of dried chlorides $\left(\mathrm{CaCl}_{2}\right.$ or $\mathrm{SrCl}_{2}$, and $\left.\mathrm{EuCl}_{3}\right)$ and sulfur. Thioglycerol was added as a capping agent and ethylenediamine was used as a solvent. The autoclave was heated and maintained for $12 \mathrm{~h}$ at a temperature of 180 to $200^{\circ} \mathrm{C}$. More details on this solvothermal synthesis 
method, as used for the production of europium-doped alkaline earth sulfide particles, can be found elsewhere $[12,13]$.

The crystallinity of the as-obtained thin films was measured with $\theta-2 \theta$ x-ray diffraction using $\mathrm{CuK} \alpha$ radiation. Pole figures were recorded with a diffractometer (Bruker D8) equipped with an Euler cradle to study the in-plane crystallographic orientation of the thin films. The morphology of the layers was studied by means of scanning (FEI Quanta 200F) and transmission electron microscopy (TEM, JEOL JEM2200FS). Luminescence was studied with a fluorescence spectrometer (FS920, Edinburgh Instruments). Photoluminescence emission spectra were obtained at an excitation wavelength of $425 \mathrm{~nm}$. Electroluminescence spectra were obtained using a sinusoidal wave form with a frequency of $1 \mathrm{kHz}$.

\section{Results and discussion}

\subsection{Luminescence}

Upon recovering the samples from the autoclave, a bright and homogeneously luminescent layer was observed under UV excitation. (Fig. 1). The bottom part of the deposited layer (Fig. 1) is characterized by an irregular, agglometrated growth of particles. The remainder of the thin film appears homogeneous, characterized by a bright, red emission colour under UV excitation. Fig. 1 shows the photoluminescence spectrum for a solvothermally grown CaS:Eu thin film having a single emission peak at $650 \mathrm{~nm}(\mathrm{FWHM}=68 \mathrm{~nm})$, which is identical to the emission in bulk material [14].

The emission spectrum of the $\mathrm{Eu}^{2+}$ ions, based on a $5 \mathrm{~d}-4 \mathrm{f}$ transition, is very sensitive to the crystal field. For instance, the emission peak shifts from $650 \mathrm{~nm}$ in CaS:Eu to 610nm in SrS:Eu. If the solvothermally grown layers would have a lot of defects or if the Eu ions would agglomerate in grain boundaries, changes in the width and/or position of the emission peak could be expected. From the similarity between bulk and thin film 


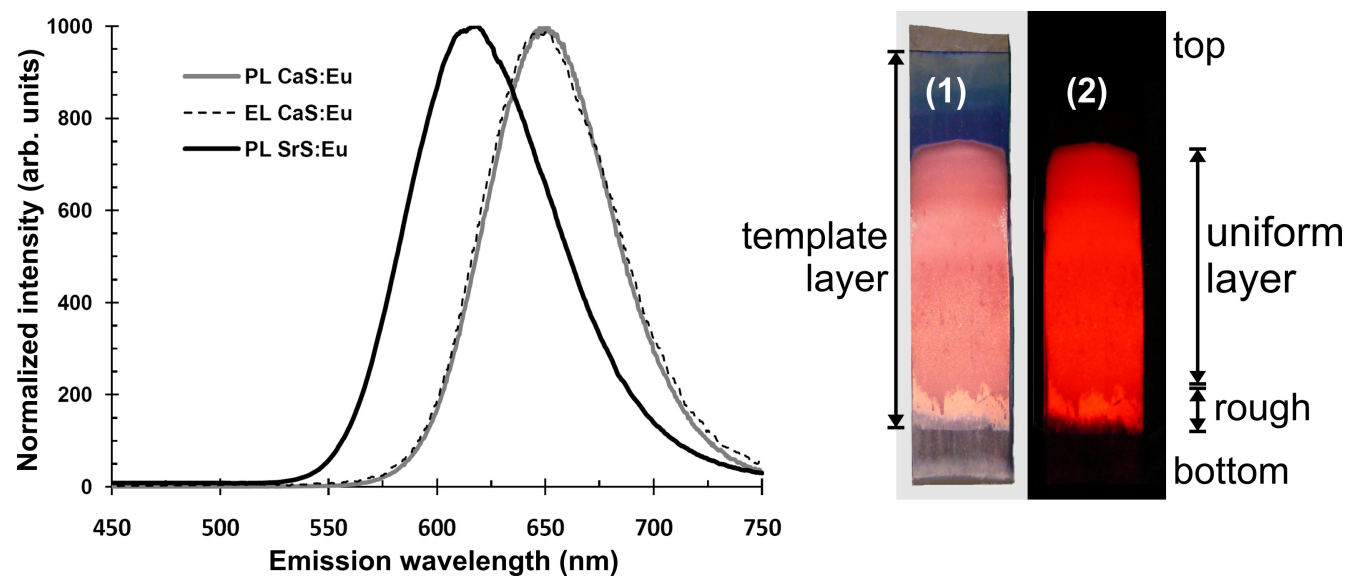

Figure 1. (left) Photoluminescence (PL) and electroluminescence (EL) spectra of solvothermally deposited SrS:Eu and CaS:Eu thin films. (right) Photograph of a solvothermally deposited CaS:Eu thin film on a templated Si substrate, under (1) UV + visible light and (2) UV only (colour online).

spectra, it can be concluded that the europium ions are well incorporated into the sulfide lattice, even without post-deposition annealing. The thin films were also electrically contacted to evaluate if the films can be used for electroluminescence applications. Fig. 1 shows the EL emission spectrum, obtained at a driving frequency of $1 \mathrm{kHz}$. This deposition technique is expected to be applicable also for the deposition of other sulfide materials. As an example, Fig. 1 shows the emission spectrum for a solvothermally grown SrS:Eu thin film. The photoluminescence peaks at $610 \mathrm{~nm}$, corresponding to an orange emission colour. The remainder of this article focusses on the growth and the morphology of CaS:Eu thin films, as similar conclusions can be drawn for SrS:Eu thin films.

\subsection{Texture and morphology}

The morphology of the CaS:Eu layers was studied with scanning electron microscopy (SEM). From the edge-on view shown in Fig. 2(a), one readily observes the strongly textured surface of the solvothermally deposited thin film. The average film thickness is 
about $2.5 \mu \mathrm{m}$, with the surface terminated by pyramid-shaped crystallites. In Fig. 2(a) the template layer is barely visible, given its thickness of only 40nm. The deposited thin film is densely packed and no voids between the crystallites are visible with SEM. Furthermore, we observed a homogeneous growth of the deposited thin film over large parts of the templated substrate (Fig.1). Depending on the synthesis conditions (template layer, capping agents), the surface can be changed from relatively flat to strongly textured, with micron-sized pyramidal shapes. Top views of two thin films with clearly different surface roughness are shown in Fig. 2(b) and (c). It was verified that the solvothermal growth does not occur on bare substrates but only on top of the template layers.
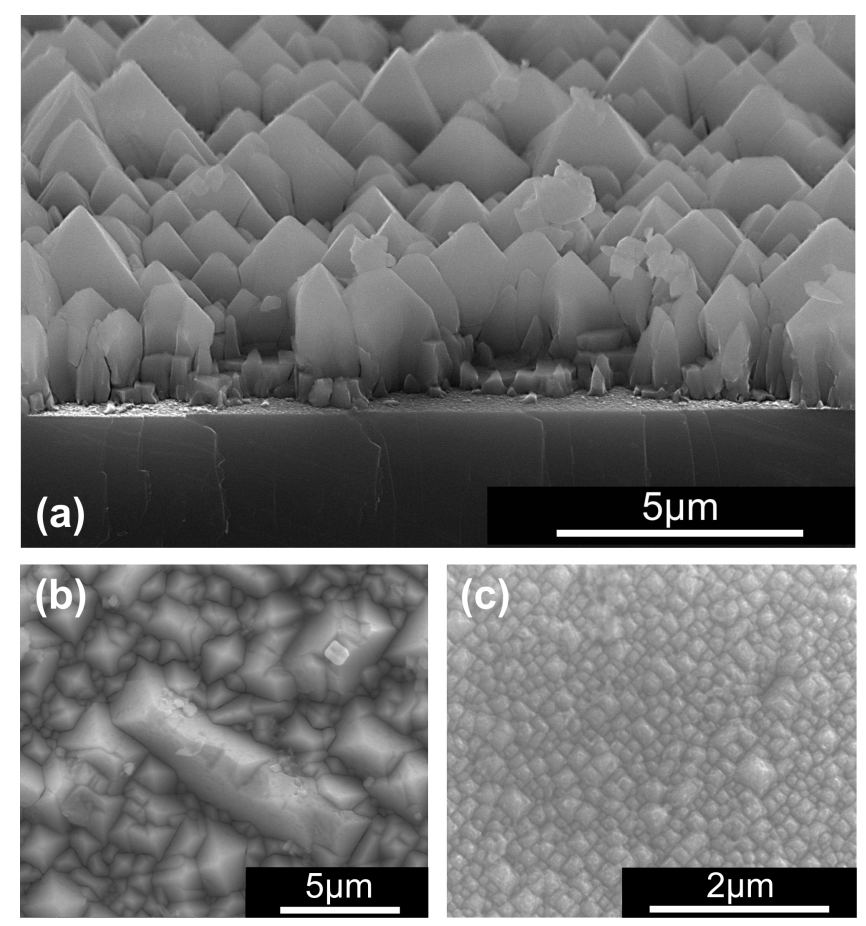

Figure 2. Scanning electron microscopy images of solvothermal CaS:Eu thin films deposited on a templated Si substrate (a) cross-section, (b) and(c) top view of two layers with different surface roughness.

In the x-ray diffraction pattern, recorded in a $\theta-2 \theta$ configuration, only the diffraction peaks belonging to the (200) planes are clearly visible (Fig. 3), both for the deposition 
on vycor and Si (100) substrates. The diffraction intensity for the (200) peak is about 250 times stronger than the one for the (220) diffraction, while this ratio should be about 1.4 for a random orientation of diffracting CaS grains. This points to a strong preferential orientation of the CaS crystallites in the solvothermally deposited layer.

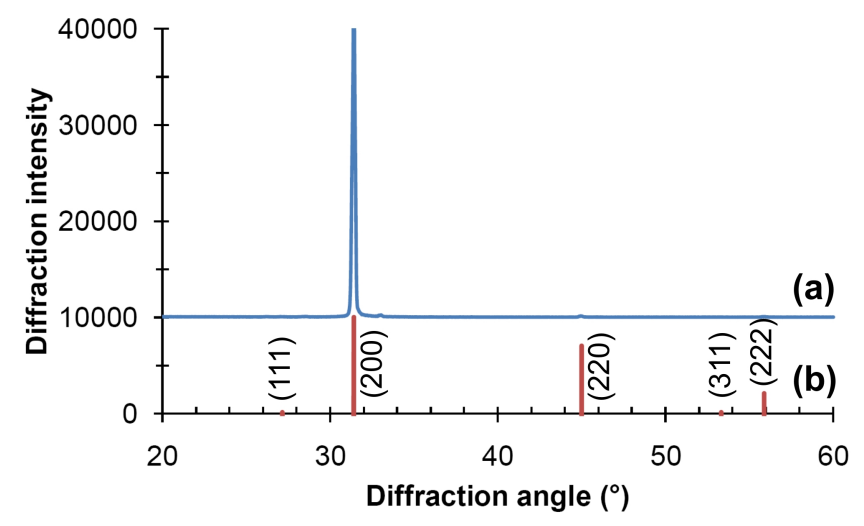

Figure 3. (a) x-ray diffraction pattern of a CaS:Eu thin film, measured in $\theta-2 \theta$ geometry. (b) ASTM reference pattern for CaS powder.

Pole figures were recorded for the (220) diffraction (Fig. 4) to further analyse the crystallographic orientation of the thin film. This confirms the preferential orientation, with all the diffraction intensity observed at a tilt angle $\chi$ of $45^{\circ}$, which is the angle between the (200) and (220) lattice planes in the cubic CaS lattice. Fig. 4(c) shows the diffraction intensity at $\chi=45^{\circ}$, depending on the in-plane rotation $\varphi$ for both types of substrates. On vycor, no preferential in-plane alignment is observed. When deposited on $\mathrm{Si}$, the pole figure consists of 4 major peaks at $\varphi=45,135,225$ and $315^{\circ}$. The FWHM of the peaks is about $8^{\circ}$ in $\varphi$ and $3^{\circ}$ in $\chi$. This strong in-plane orientation is explained by the almost epitaxial growth of CaS $(\mathrm{a}=569.5 \mathrm{pm})$ onto $\mathrm{Si}(543.1 \mathrm{pm})$. The epitaxial growth is plausible given the small $(4.8 \%)$ difference in lattice constants. This strong texturing is also observed upon inspection of the thin film surface in SEM, as the majority of the pyramid-shaped grains possesses the same orientation within a few degrees. Next to this epitaxial growth, 4 minor peaks (at $\varphi=0,90,180$ and 
$270^{\circ}$ ) are observed in the $(220)$ pole figure. These minor peaks mainly originate from the secondary growth of (small) crystallites at the boundary of two epitaxially grown crystallites.

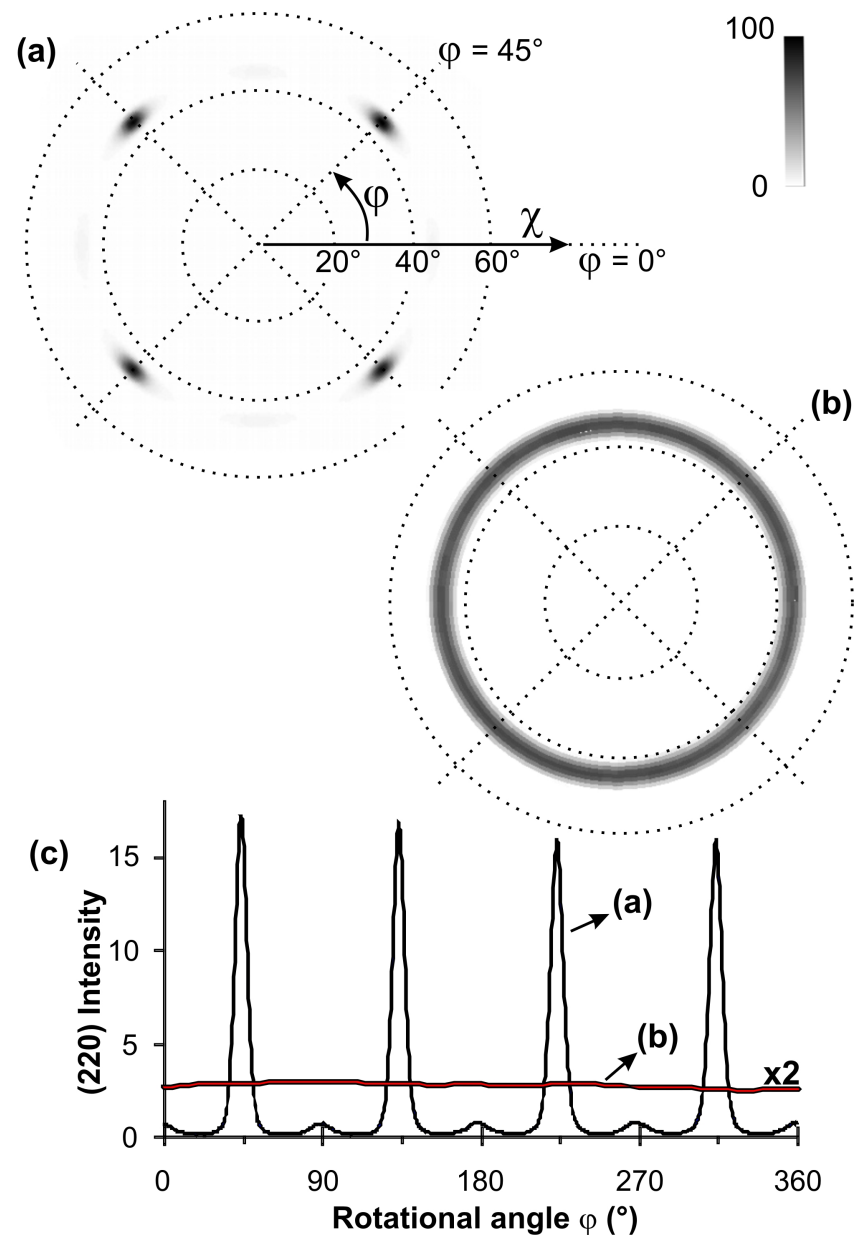

Figure 4. (220) pole figure of a CaS:Eu thin film on (a) silicon and (b) vycor. (c) Diffraction intensity at $\chi=45^{\circ}$ for the pole figures (a) and (b).

The TEM image of the cross-section of a solvothermally grown CaS:Eu thin film on a Si substrate (Fig.5(a)) reveals large grains extending from the template layer to the surface of the thin film. The selected area diffraction pattern (Fig. 5(b)) confirms the single-crystalline character of the grains, with the [100] zone axis perpendicular to the image in this case. The microstructure of the template layer was studied by TEM and it appeared that it consists of relatively small grains, with a orientational bias of 
the (200) planes parallel to the Si substrate surface. During the solvothermal deposition process grains with this orientation overgrow grains with deviating orientation to arrive at an almost fully oriented thin film (Fig. 4).

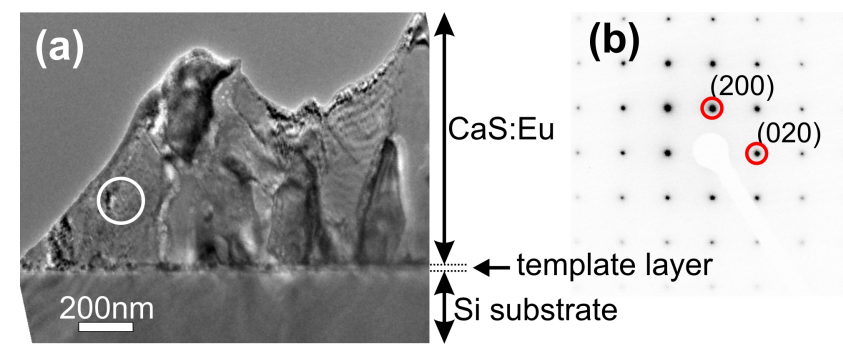

Figure 5. (a) TEM cross-section of solvothermal CaS:Eu thin films, (b) selected area diffraction pattern of the area encircled in (a).

\subsection{Localized growth}

As mentioned before, the solvothermal growth process only occurs on top of template layer. To check the possibility of a localized solvothermal deposition of the sulfide thin film, the following procedure was used. On top of a silicon substrate with an undoped CaS template layer, $2 \mathrm{~mm}$ circular dots of $\mathrm{Al}_{2} \mathrm{O}_{3}$ (thickness of $50 \mathrm{~nm}$ ) were deposited by electron-beam evaporation using a mask. Then, the substrate was loaded into the autoclave and the solvothermal process was applied. Fig. 6 shows a SEM image and EDX elemental maps, which confirms solvothermal growth only occurring on top of the bare template layer, and not on top of the $\mathrm{Al}_{2} \mathrm{O}_{3}$ layer. Fig. 6(b) shows the sharp edge between the solvothermally grown thin film and the $\mathrm{Al}_{2} \mathrm{O}_{3}$ mask layer. Hence it can be expected that a localized growth can be obtained after patterning of the template layer. Then, during the solvothermal deposition process, growth will only occur in the desired areas, which is a technological advantage compared to post-deposition etching. 

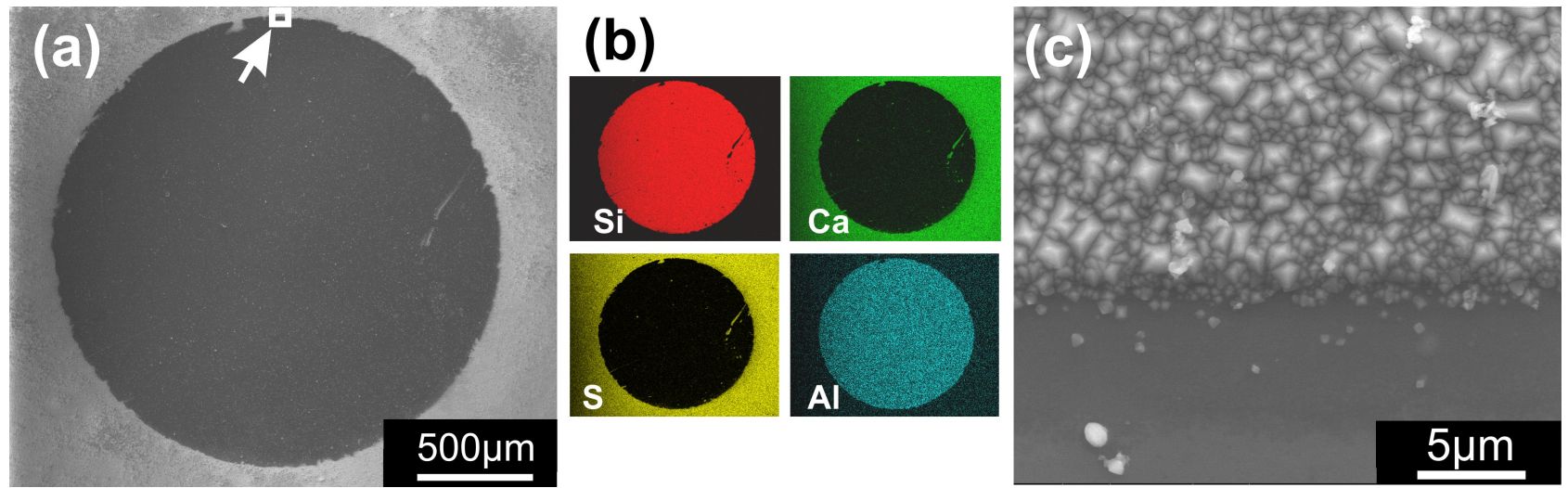

Figure 6. (a) SEM image of a solvothermally grown CaS:Eu thin film on top of an $\mathrm{Al}_{2} \mathrm{O}_{3}$-masked templated substrate. (b) Elemental maps of sulfur, aluminum, silicon and calcium as obtained by EDX on the area shown in (a). (c) detailed image of the edge as indicated by the arrow in (a) [colour online].

\section{Conclusions and perspectives}

We presented a method to deposit luminescent and strongly crystalline sulfide thin films with a non-vacuum technique onto templated substrates. Furthermore, the deposition temperature is limited to $200^{\circ} \mathrm{C}$ and no post-deposition thermal treatment is required. The thin films are strongly textured and show a preferential (200) out-of-plane orientation. On silicon substrates, an almost epitaxial growth was obtained.

The low temperature route could pave the road for deposition onto flexible substrates. Also, non-vacuum routes for the template layer will be explored to eventually arrive at an entirely non-vacuum deposition technique. In any case we have already shown that high quality thin films can be grown onto e-beam deposited template layers of relatively poor crystallinity.

Acknowledgments. One of the authors (PFS) is a post-doctoral research fellow of FWO-Vlaanderen. 
Textured CaS:Eu thin films by a solvothermal process 
[1] P. F. Smet, D. Poelman, and R. L. Van Meirhaeghe, J. of Appl. Phys. 95 (2004) 184-190

[2] D. Poelman, J.E. Van Haecke, P.F. Smet, J Mater Sci: Mater Electron 20 (2009) S134-S138

[3] M.Z. Yang, J. Moore, X.W. Wu, ASID'04: Proceedings of the $8^{\text {th }}$ Asian Symposium on Information Display (2004) 275-277

[4] P. F. Smet, K. Korthout, J. E. Van Haecke, and D. Poelman, Mat. Sci. Eng. B 146 (2008) 264-268

[5] R.D. Dupuis, M.R. Krames, Journal of lightwave technology 26 (2008) 1154-1171

[6] E.F. Schubert, J.K. Kim, H. Luo, et al. Reports on Progress in Physics 69 (2006) 3069-3099

[7] R. Mueller-Mach, G. Mueller, M.R. Krames, H.A. Hoppe, F. Stadler, W. Schnick, T. Juestel, P. Schmidt, Phys. stat. sol. A 202 (2005) 1727-1732

[8] D. Poelman, and P.F. Smet, J. Phys. D: Appl. Phys. 36 (2003) 1850-1857

[9] J.E. Van Haecke, P.F. Smet, D. Poelman, J. Electrochem. Soc. 152 (2005) H225-H228

[10] H.H. Shin, J.H. Kim, B.Y. Han, J.S. Yoo, Jpn. J. Appl. Phys. 47 (2008) 3524-3526

[11] K. Onisawa, M. Fuyama, K. Taguchi, K. Tamura, and Y. A. Ono, J. Electrochem. Soc. 135 (1988) 2631-2634

[12] J. E. Van Haecke, P. F. Smet, K. De Keyser, and D. Poelman, J. Electrochem. Soc. 154 (2007) J278-J282

[13] C. R. Wang, K. B. Tang, Q. Yang, C. H. An, B. Hai, G. Z. Shen, and Y. T. Qian, Chem. Phys. Lett. 351 (2002) 385-390

[14] P. F. Smet, J. E. Van Haecke, F. Loncke, H. Vrielinck, F. Callens, and D. Poelman, Phys. Rev. B $74(2006) 035207$ 